\title{
El proceso de urbanización en la cuenca del río Chillán y su capacidad adaptativa ante precipitaciones extremas
}

\section{The process of urbanization in the Chillán's watershed Chillán and its adaptive capacity to stormwater}

\author{
Cristian Henríquez Ruiz*
}

\section{INTRODUCCIÓN}

La ciudad de Chillán presenta una gran importancia histórica en el proceso de colonización de Chile Central y también por ser un polo tradicional de producción agropecuaria. Sin embargo también se destaca por ser escenario de una serie de desastres naturales, esencialmente terremotos e inundaciones, que la han destruido total o parcialmente durante el transcurso de su historia, obligando a las autoridades y habitantes a cambiar su emplazamiento en varias oportunidades y reconstruirla en otras tantas.

Tanto las fuerzas endógenas como exógenas han acompañado al proceso de urbanización, y seguirán siendo un elemento determinante en la configuración urbana futura. Para enfrentar estos desafíos y en especial a los vinculados a las consecuencias de precipitaciones extremas, como inundaciones y anegamientos, es fundamental contar con una visión integrada que reconozca a la cuenca hidrográfica como unidad de planificación territorial.

En general, el proceso de planificación chileno se ha destacado por la escasa consideración de los efectos de la urbanización en los sistemas hídricos y la falta de integración de éstos a la ciudad, transformando su cauce y riberas

\footnotetext{
* Instituto de Geografía, Pontificia Universidad Católica de Chile.
} 
en sitios eriazos; depósitos de basuras, escombros y residuos líquidos; espacios inseguros y barreras socio espaciales; y esencialmente espacios de alta vulnerabilidad ante eventos de crecidas extremas. Y también por ser muy permisible en la impermeabilización de zonas sensibles.

Uno de los más notables impactos de la urbanización sobre el sistema ecohidrológico es el aumento del flujo de escorrentía superficial, ya que el reemplazo de las coberturas naturales por coberturas impermeables, tienden a incrementar el nivel de escorrentía en comparación con las áreas naturales y rurales. El aumento del escurrimiento es especialmente crítico ante los flujos provocados por tormentas invernales extremas, en que la lluvia se concentra en pocas horas, generando inundaciones en los sectores topográficamente más deprimidos, destrucción de viviendas de condiciones estructurales precarias, colapso de red de alcantarillas y red de desagüe de aguas lluvias, erosión de márgenes de los esteros y canales, y también afecta la calidad del agua (Melesse y Wang, 2007).

Por otro lado, las cuencas como áreas geográficas son unidades óptimas de organización para conducir el manejo del agua y los recursos relacionados, pero los límites naturales de las cuencas raramente coinciden con jurisdicciones políticas y así ellas pueden no ser muy útiles para propósitos políticos, institucionales y de financiamiento (Parra, 2000), como es el caso de una planificación territorial sostenible.

En este contexto y considerando que la cuenca de Chillán ha experimentado cambios importantes en el último tiempo se plantean como objetivos:

- Analizar los cambios en los usos/coberturas de suelo experimentados en la cuenca del río Chillán en el período 1976-2006 y los cambios en la escorrentía superficial

- Describir el comportamiento de las precipitaciones extremas que generan escorrentía y los efectos de éstas en las inundaciones, anegamientos y daños en la ciudad de Chillán.

La estructura de esta comunicación se organiza en cuatro secciones: en la primera se revisa el contexto histórico de los riesgos naturales en Chillán y conceptos teóricos que sustentan la investigación, luego se presenta el área de estudio y metodología, los resultados de los objetivos planteados se abordan en la tercera parte, para terminar con algunas conclusiones e interrogantes finales. 


\section{CHILlÁN, CIUDAD DE CATÁSTROFES}

A Chillán también se le conoce como la ciudad de las cuatro fundaciones, ya que luego de su fundación en 1580 le suceden otras tres que implicaron un cambio del emplazamiento original. La ciudad de Chillán fue fundada por el Gobernador Don Martín Ruiz de Gamboa el 25 de junio de 1580 bajo el título de San Bartolomé de Chillán y Gamboa, sobre la base de un pequeño fuerte levantado el 18 de marzo de 1579 por el propio Mariscal bajo las órdenes del Gobernador Don Rodrigo de Quiroga, junto al río Chillán en unas vegas llamadas El Bajo (Asta-Buruaga, 1899).

Chillán en un comienzo fue atacada por los indios chiquillanes, de los que procede su nombre: "silla del sol". Además de la función defensiva, como la mayoría de los asentamientos fundados en la región, la ciudad se emplazaba en Camino Real que unía las ciudades de Santiago y Concepción (Concha, 1981).

"La fundación de esta ciudad tenía por objeto mantener en quietud a los indios de la región bañada por el río Itata e impedir que los rebeldes del sur pudieran comunicarse con los habitantes del lado norte de ese río" (Barros-Arana, 1884: p.12).

En 1620 fue atacada por el cacique Lientur y en 1655 por el cacique Quelantaru (Concha, 1981). El 15 de marzo de 1657 sucedió un terremoto que demolió la ciudad, iniciándose en 1664 la segunda refundación en un nuevo sitio (Rebolledo et al., 2002). En los inviernos de 1679, 1735 y 1748, se producen desastrosas inundaciones por la crecida del río Chillán.

El 25 de mayo de 1751 se produce un violento terremoto que junto a una inundación extraordinaria del río Chillán, determinaron la inmediata decisión de trasladar la ciudad, correspondiendo a la tercera refundación. Se estima que la magnitud de este evento fue de $8,5^{\circ}$ en escala Richter, ocasionando una víctima fatal (Mardones, 1990). Ese año el Gobernador Don Ortiz de Rozas funda la nueva ciudad en el sitio llamado Alto de la Horca, actual Chillán Viejo.

Posterior al período de la Colonia, y ya en la fase de consolidación de la Independencia del dominio español, sucede otro hito histórico que representa la cuarta refundación de Chillán, el cual corresponde al gran terremoto del 20 de febrero de 1835. La magnitud del evento se calcula varió entre 8 a $8,2^{\circ}$ en escala Richter, se registraron 8 víctimas fatales y Chillán fue casi completamente destruido, salvándose solo algunas casas (Mardones, 1990). Este evento obligó al Presidente Joaquín Prieto a fundar el actual Chillán, disponer el traslado de Chillán Viejo y restringir la urbanización en esta área. Esta dis- 
posición no fue acatada por los vecinos de menores recursos de Chillán Viejo, creciendo ambas ciudades simultáneamente. Como consecuencia de esta disposición legal se encuentra la estructura urbana casa huerto familiar o casaquinta en Chillán Viejo, que ha permanecido a lo largo del tiempo. En 1895 Chillán y Chillán Viejo concentraban 28.738 habitantes (Rebolledo, et al., 2002).

Durante el siglo xx, el 24 de enero de 1939 se produce otro violento terremoto pereciendo 5.685 personas y el $60 \%$ de las viviendas y edificios se derrumbaron o quedaron semi destruidos (Urrutia y Lanza, 1993), transformándose en uno de los más trágicos de la historia del país. Estudios citados por Mardones (1990) indican que el evento se prolongó por 3 minutos registrando una magnitud de $8,3^{\circ}$ en escala Richter y $11^{\circ}$ en Mercalli. En la provincia de Biobío también se hizo sentir los efectos del movimiento telúrico registrándose 36 muertos y numerosos heridos (Urrutia y Lanza, 1993). Si bien este evento no significa una quinta relocalización, este desastre natural tiene un fuerte impacto en el crecimiento poblacional, ya que además de producir un número importante de muertes directas, como consecuencia indirecta se generan emigraciones hacia otras ciudades por el temor de la población a otra catástrofe de esta envergadura. Mientras que desde un punto de vista económico, a partir de este hito, junto al Plan Chillán de la década de 1950 e innovaciones tecnológicas en las actividades agrícolas, se impulsa su desarrollo y crecimiento (Reyes, 1990).

En 1953 se produce otro violento sismo que afectó Chillán, pereciendo 5 personas y muchos daños materiales. Ese mismo año se produce un nuevo temporal en la región por tres días, donde los más afectados fueron las barriadas populares en Chillán, donde se rebalsaron varios canales (Urrutia y Lanza, 1993).

En mayo de 1960 sucede un nuevo terremoto con epicentro en la ciudad de Valdivia que devasta el país, ocasionando 5 decesos en Chillán y destruyendo un $20 \%$ de las viviendas.

De 1970 en adelante destacan los frentes de mal tiempo de 1974, 1975, 1982, 1984, 1986 y 1991 que afectaron a la VIII región del Biobío y también a la ciudad de Chillán, ya que se desbordaron la mayoría de los ríos, esteros y canales, ocasionando daños en las viviendas, damnificados y anegamientos de terrenos cultivables (Urrutia y Lanza, 1993).

Ya en la actualidad, uno de los eventos más caóticos sucedió el año 2006, entre los días 11 y 12 de julio de 2006, donde se produjo una precipitación extrema que afectó fuertemente la zona centro sur del país, en especial la región del Biobío, el cual originó graves inundaciones, anegamientos, deslizamientos 
y desbordes de cauces. Este episodio pluviométrico, que llevó a las autoridades a declarar "Zona de Catástrofe" a la región, afectó a la población más vulnerable, registrándose numerosas pérdidas de vidas humanas, miles de damnificados y grandes costos económicos en viviendas e infraestructura. En el caso específico de Chillán los sectores más afectados corresponden al estero Las Toscas y en especial los canales que cruzan la ciudad, los cuales colapsaron e inundaron grandes sectores urbanos, al igual que el sistema de evacuación de aguas lluvias.

A partir de esta ajustada síntesis se puede aducir que los riesgos naturales, principalmente los terremotos y las inundaciones, han sido un aspecto co-sustancial en el crecimiento histórico de la ciudad, el cual seguirá siendo determinante en el crecimiento futuro. Aprender a vivir con estos riesgos implica un importante desafío para poder disminuir la vulnerabilidad y adaptarse a estas condiciones ambientales.

\section{Vulnerabilidad, resiliencia y capacidad adaptativa ante perturbaciones naturales}

Antes de adentrarse a los resultados es importante detenerse brevemente en algunos conceptos que contextualizan la investigación. Es ampliamente conocido que no existe riesgo sin vulnerabilidad, así como también no existe vulnerabilidad sin la presencia de una amenaza. La vulnerabilidad en términos simples se entiende como el grado de susceptibilidad ante una perturbación, daño, peligro o golpe (shock). Son componentes de la vulnerabilidad según Gallopín (2006): la sensitividad (capacidad de un sistema humano o natural para absorber impactos sin sufrir en el largo plazo daños o cambios significativos), la capacidad de respuesta (habilidad de ajustarse a los disturbios) y la exposición (grado, duración y/o extensión en la cual un sistema está en contacto, o sujeto a una perturbación). Estos conceptos se pueden entender fácilmente con el ejemplo que da este autor en relación a las inundaciones en una comunidad: los hogares más precarios son golpeados más fuerte que los hogares que poseen casas sólidas (sensitividad), a menudo los hogares más pobres están localizados en lugares más susceptibles a las inundaciones (exposición), por otro lado, las familias de mayores recursos tienen mayor disponibilidad de medios para reparar los daños (capacidad de respuesta). Por lo tanto la magnitud final del impacto depende de la intensidad, magnitud y permanencia de la inundación los cuales son atributos de la perturbación. 
Un concepto interesante para entender la vulnerabilidad es el de resiliencia, que se podría entender como su antónimo. Este fue introducido por Holling (1973) desde el campo de la ecología y originalmente fue definido como la medida de persistencia de un sistema y de sus habilidades para absorber los cambios y perturbaciones para mantener las mismas relaciones entre las poblaciones y variables esenciales. Actualmente existen muchas definiciones del concepto, desde perspectivas netamente ecológicas a otras con gran énfasis en lo social, pasando por interpretaciones más híbridas que integran ambos enfoques (Brand y Jax, 2007; Gallopín, 2006; Walker, et al., 2004).

Desde esta perspectiva surge el concepto de resiliencia socio-ecológica, entendido como la capacidad de un sistema socio-ecológico para absorber los disturbios recurrentes, así como retener las estructuras, procesos y feedback esenciales (Adger, et al., 2005). Tomando esta definición como marco de referencia, se puede considerar a la cuenca hidrográfica como un sistema socioecológico (Gallopín, 2006) en la cual se conjugan distintos subsistemas antrópicos y naturales que interactúan de manera recíproca a distintas escalas temporales y espaciales. Las cuencas donde interactúan usos de suelos urbanos, agrícolas y naturales ilustran a este tipo de sistema, A su vez las cuencas hidrográficas, en el caso de Chile Central, son afectadas por disturbios recurrentes como lo son las precipitaciones extremas, propias del clima mediterráneo, que normalmente ocasionan importantes inundaciones, anegamientos ${ }^{1} \mathrm{y}$ deslizamientos.

Periódicamente en la cuenca de Chillán se han registrado montos de precipitaciones extremas de carácter extraordinario que han destruido y producido graves consecuencias en la infraestructura física y pérdida de vidas humanas, por lo que se podría decir que no han operado los mecanismos de resiliencia del sistema que permitan reponerse a estas perturbaciones. O en otros términos conceptuales, y probablemente más preciso, se ha observado una baja capacidad adaptativa.

Este último concepto (también llamado adaptabilidad) al igual que el de resiliencia nace en las ciencias naturales (Smith y Wandel, 2006), refiriéndose a la habilidad de llegar a ser adaptado, esto es la capacidad de vivir y reproducirse, en una cierta gama de contingencias ambientales. La capacidad de adap-

\footnotetext{
${ }^{1}$ Inundación se entiende como el desastre o catástrofe vinculada a la acumulación o flujo descontrolado de agua superficial en zonas donde existes asentamientos o actividades humanas. El anegamiento se relaciona con la acumulación superficial de las aguas lluvias o con el afloramiento de la napa subterránea, sin que necesariamente intervenga la acción de un flujo de escurrimiento lineal (Mardones, 1990).
} 
tarse implica mejorar las condiciones de una especie, población o individuo, en su ambiente, incluso ante la ausencia de cambios. Lo relevante es que también se aplica para los sistemas humanos, donde la capacidad de aprendizaje y progresos tecnológicos, aseguren una viabilidad de las actividades económicas y sociales, y una calidad de vida. De esta forma y siguiendo a Gallopín (2006: p. 300), la capacidad adaptativa se puede definir como: la capacidad de cualquier sistema humano, desde un individuo hasta toda la humanidad, de incrementar (o al menos mantener) la calidad de vida de sus miembros en un ambiente dado. En términos generales la capacidad adaptativa parece ser un concepto más amplio e integrador que la capacidad de respuesta, y vinculado directamente con la sostenibilidad, por lo que se considera apropiado como marco de referencia para esta investigación.

\section{Metodología}

\section{Área de estudio}

La cuenca del río Chillán se ubica entre los $71^{\circ} 00^{\prime}$ y $72^{\circ} 30^{\prime}$ de longitud oeste y los $36^{\circ} 30^{\prime}$ y $37^{\circ} 00^{\prime}$ de latitud sur, tiene una superficie de drenaje de $822,7 \mathrm{~km}^{2}$, cifra que representa el $7,4 \%$ de la cuenca del Itata que cubre un área de $11.090 \mathrm{~km}^{2}$ (Niemeyer y Cereceda, 1984). El río Chillán tiene una extensión de $105 \mathrm{~km}$ en sentido SE-NW, naciendo en la falda poniente de Los Nevados del Chillán en la Cordillera de Los Andes, a unos 3.200 metros de altitud, y confluye en el río Ñuble que es la mayor arteria del sistema Itata, a 75 msnm en la depresión intermedia.

Administrativamente pertenece a la VIII región del Biobío, provincia del Nuble y a nivel comunal comprende la mayor parte de los municipios de Chillán, Chillán Viejo, Pinto y Coihueco y una pequeña porción de la comuna de San Ignacio (figura 1).

El clima de la parte central de la cuenca de Chillán se caracteriza por ser templado cálido, con estación seca y lluviosa semejante, determinado por el movimiento latitudinal del Anticiclón del Pacífico. El monto de precipitación anual bordea los $1.050 \mathrm{~mm}$, concentrándose entre mayo y agosto el $67,9 \%$ del agua caída. El régimen pluviométrico invernal se asocia a las perturbaciones del frente polar que traslada centros de baja presión hacia el continente mediante vientos con dirección W a E y S a N (Mardones, Echeverría y Jara, 2005). De acuerdo al perfil pluviométrico trazado a los $36^{\circ} 45^{\prime}$ S incluido en el estudio de Mardones et al (2001), existe un gradiente positivo de precipi- 


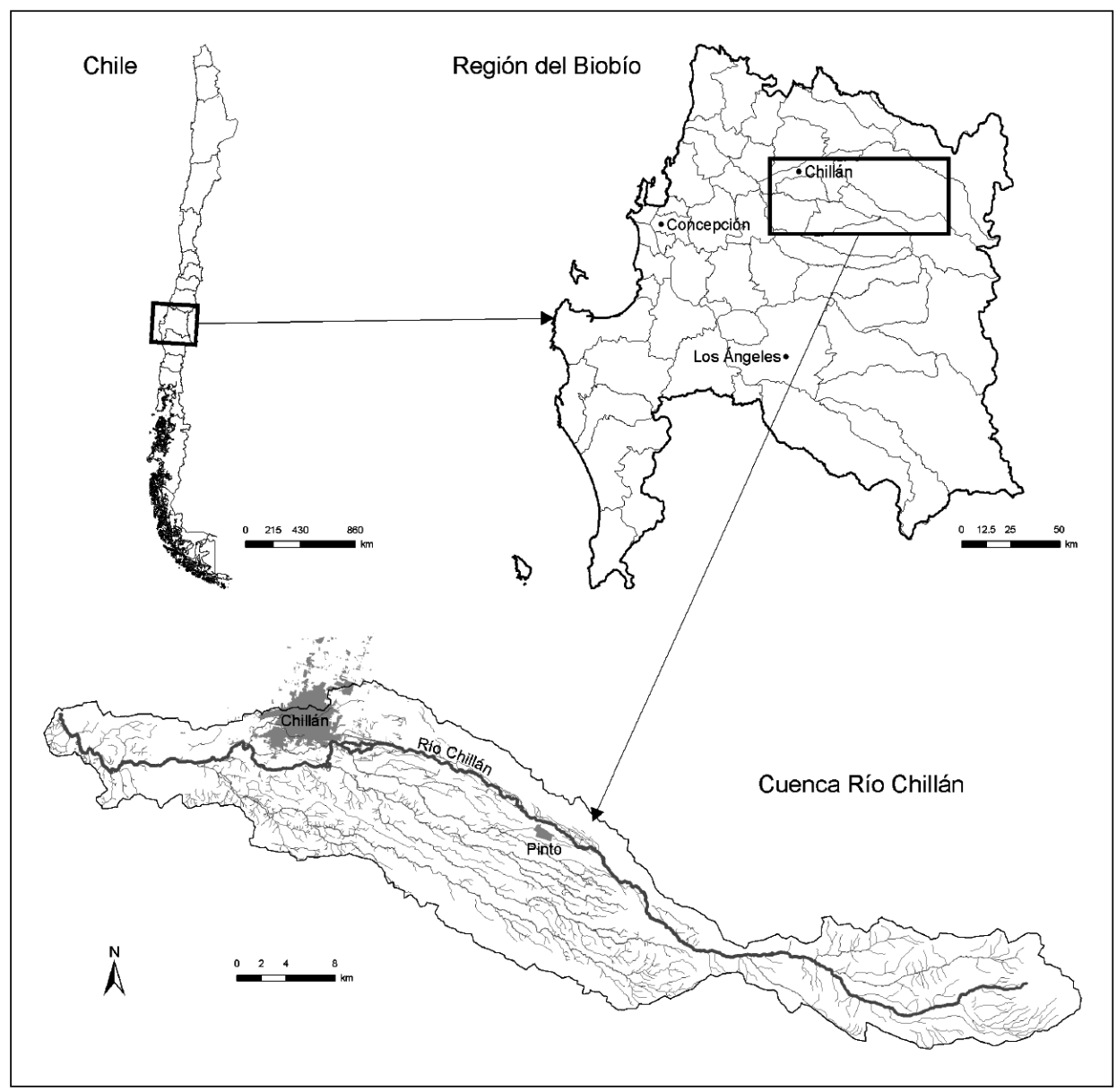

Figura 1. Localización de la cuenca de Chillán

tación a medida que se asciende en altitud hacia el este, de esta forma en las laderas de barlovento de la Cordillera de Los Andes se registran montos pluviométricos entre 2.500 y $3.000 \mathrm{~mm}$.

La ciudad de Chillán desde un punto de vista geomorfológico se emplaza sobre la Formación Mininco de origen Pliocénico. La cuenca en este sector está profundamente deprimida lo cual ha permitido la recepción de toda clase de acarreos provenientes de los macizos andinos, gruesos en el borde oriental de la depresión intermedia y predominantemente finos hacia el sector occiden- 
tal. La presencia de la Cordillera de la Costa se presentó muy ventajosa para este proceso de acumulación, ya que sirvió como una barrera de contención a los sedimentos, originando la presencia de anegamientos y defluviaciones temporales, como es posible evidenciar en los paleocanales existentes donde se observan sedimentos limo-arcillosos (Mardones, 1990).

Los suelos de la cuenca presentan buenas características de drenaje, así como bajos niveles de erosión como se aprecia en el Cuadro 1. Igualmente hay una concentración importante de suelos con aptitud agrícola (Clases I a IV) por sobre el $50 \%$, los cuales se distribuyen principalmente en la parte central de la cuenca.

CUADRO 1.

CARACTERÍSTICAS DE LOS SUELOS EN LA CUENCA DE CHILLÁN

\begin{tabular}{|c|c|c|c|c|c|c|c|c|}
\hline \multicolumn{3}{|c|}{ Drenaje } & \multicolumn{3}{|c|}{ Fragilidad } & \multicolumn{3}{|c|}{ Capacidad de uso } \\
\hline Tipo & Sup. (ha) & $\%$ & Tipo & Sup. (ha) & $\%$ & Clases & Sup. (ha) & $\%$ \\
\hline Muy pobre (1) & 498,6 & 0,6 & Ligera (1) & $8.692,8$ & 10,6 & I & 154,8 & 0,2 \\
\hline Pobre (2) & 682,6 & 0,8 & Moderada (2) & $6.491,2$ & 7,9 & II & $21.628,7$ & 26,3 \\
\hline Imperfecto (3) & $6.550,7$ & 8,0 & Severa (3) & $2.329,9$ & 2,8 & III & $10.658,5$ & 13,0 \\
\hline Moderado (4) & $3.568,1$ & 4,3 & Sin erosión (4) & $45.751,0$ & 55,6 & IV & $11.794,6$ & 14,3 \\
\hline Bueno (5) & $52.820,5$ & 64,2 & Sin información & $19.006,7$ & 23,1 & VI & $6.855,6$ & 8,3 \\
\hline Excesivo (6) & $4.990,0$ & 6,1 & & & & VII & $13.998,2$ & 17,0 \\
\hline Sin información & $13.162,8$ & 16,0 & & & & VIII & $2.472,3$ & 3,0 \\
\hline & & & & & & Sin información & $14.709,9$ & 17,9 \\
\hline
\end{tabular}

Nota: La categoría sin información corresponde a la parte cordillerana de la cuenca y coberturas urbanas. Fuente: Elaboración propia en base a datos de CIREN.

Dentro de los estudios relacionados con inundaciones y anegamientos en la cuenca y ciudad de Chillán se pueden mencionar: el "Plan Maestro de Manejo de Cauces, Cuenca del Río Chillán, VIII Región" (PRISMA, 2004), el "Plan Maestro de Evacuación y Drenaje de Aguas Lluvias de Chillán" (INGENDESA, 2002), el estudio sobre riesgos naturales en la ciudad de Chillán (Mardones, 1990) y el "Plan Regulador Intercomunal Chillán-Chillán Viejo" (GORE-BIOBIO, 2007). Dentro de los principales resultados destaca el diagnóstico de riesgo por inundación planteado por PRISMA (2007: pp. 24-25) para la cuenca: 
- El sector puente El Saque-Las mariposas, ubicado al sur-oriente de la ciudad, corresponde al más relevante, desde el punto de vista del comportamiento del cauce en crecidas y sus efectos sobre zonas pobladas. Se caracteriza por condiciones de lecho móvil y patrón de escurrimiento trenzado. Tiene una pendiente media cercana al 0,6\%, lo que representa una disminución importante con respecto a la del tramo superior (sector Mariposas-Esperanza), perdiendo capacidad de arrastre y acumulándose material granular en el cauce. Uno de los puntos más críticos es el sector El Emboque, en él el río se encuentra muy próximo al camino ChillánTanilvoro, construyéndose por parte del Ministerio de Obras Públicas (MOP) varias defensas fluviales y obras de manejo de cauce. Además se han detectado intervenciones no autorizadas del cauce por propietarios ribereños y canalistas afectados, quienes han construido pretiles de defensa para proteger terrenos y canales en ambas riberas. Un problema particular lo constituye un brazo del río que se abre hacia la ribera derecha, pero que actualmente no está activo y sólo actúa como drenaje de aguas lluvias y derrames de riesgo.

Otro antecedente destacable es el aportado por Mardones (1990: p. 25) en cuanto a problemas de drenaje de los suelos e inestabilidad de éstos ante riesgo sísmico, especialmente en la periferia de Chillán, ya que corresponde a suelos que se saturan con facilidad como consecuencia de una alta concentración pluviométrica. Ello permite concluir que "el sitio de Chillán presenta rasgos particularmente deficientes para el asentamiento urbano".

\section{Materiales y Métodos}

El quehacer metodológico se dividió en dos grandes tareas: la determinación del cambio de uso de suelo, y la estimación de la escorrentía potencial y comportamiento de precipitaciones extremas. Destaca la ayuda de los Sistemas de Información Geográfica (SIG) y percepción remota en la obtención de resultados.

Respecto a lo primero, los cambios de usos/coberturas de suelo de la cuenca del río Chillán se determinaron mediante clasificación manual de imágenes satelitales Landsat TM, para los años 1989 (8 de marzo), 1999 (18 de noviembre) y 2007 (23 de febrero), utilizando las bandas espectrales 4-3-2, mediante los programas PCI Geomatics 9.1 y ArcGIS 9.2. También se interpretó una imagen Landsat MSS del año 1976 (18 de febrero) mediante similar técnica, la cual fue 
obtenida gratuitamente de la página web, http://www.dgi.inpe.br/. Previamente las imágenes fueron georreferenciadas utilizando puntos de control extraídos de las cartas topográficas del Instituto Geográfico Militar (IGM), escala 1:50.000 previamente escaneadas y georreferenciadas en el programa ArcGIS 9.2, utilizando el Datum WGS84, Huso 19 Sur. Se ha empleado un tamaño de celda de $25 \mathrm{~m}$.

Los usos de suelo identificados corresponden a: usos urbanos, agrícolas, matorral/pastizal, plantaciones forestales, bosque nativo, nieve, suelo desnudo y cuerpos de agua. Para precisar el uso urbano de la ciudad de Chillán se ha empleado una imagen Ikonos del día 15 de marzo del año 2006 y fotos aéreas blanco y negro del Servicio Aerofotogramétrico (SAF) del año 1991, escala 1:40.000, insumos que ofrecen una resolución espacial mucho mayor a la imagen Landsat, con lo cual fue posible discriminar el uso urbano de baja densidad en la zona periurbana.

Para validar la clasificación de uso de suelo se han empleado fotografías aéreas color del año 1998, escala 1:115.000, obtenidas de la página web http://www.sinia.cl, coberturas digitales de drenaje y caminos, y terrenos de verificación realizados el mes de febrero de 2007 y 2008.

En cuanto a lo segundo, para determinar la escorrentía superficial potencial se escogió el método del Soil Conservation Service (SCS) Technichal Release No 55 (TR 55) o también conocido como método de abstracción SCS (USDA, 1986), el cual se considera más exacto que el método racional para grandes cuencas urbanas ya que, en palabras de Randolph (2004), toma en cuenta más factores e involucra menos enjuiciamientos por parte del usuario, particularmente en la elección de los tiempos de concentración. Este método si bien es más simple que muchos modelos hidrológicos complejos como: el HSPF (Hydrological Simulation Program-Fortran del USGS), SWMM (Storm Water Management Model de USEPA), HEC-RAS (Hydrologic Engineering Centers River Analysis System de USACE) o el SAIH (Sistemas Automáticos de Información Hidrológica) ${ }^{2}$, el TR 55 es ampliamente usado en programas de control de precipitaciones de tormenta y de control de erosión y sedimentos a nivel local y regional, principalmente en Estados Unidos.

El TR 55 se basa en la teoría del destino de las precipitaciones máximas en 24 horas, en función de los resultados de estudios empíricos de varias cuencas pequeñas en Estados Unidos (Chow, Maidment y Mays, 1994; Mockus, 1972;

\footnotetext{
2 Para mayor información consultar el texto Environmental Land Use Planning and Management (Randolph, 2004) y Sistemas de ayuda a la explotación de un SAIH (Aldana, 1998). 
Randolph, 2004). Al modificar los usos y coberturas de suelo en una cuenca de drenaje se modifican los peak de descarga, por lo cual esta técnica ofrece grandes ventajas para predecir los efectos de la escorrentía superficial en distintos escenarios de uso de suelo (Henríquez, Azócar y Aguayo, 2006; Randolph, 2004).

Estos preceptos se resumen en la siguiente ecuación de escorrentía superficial:

$$
P_{e}=\frac{(P-0,2 S)^{2}}{(P+0,8 S)}
$$

Donde: $P_{e}$ es la escorrentía de tormenta; $P$ es la precipitación de tormenta; y $S$ es el almacenamiento máximo potencial en milímetros. Mientras mayor sea $S$ menor es la escorrentía superficial. Por su parte, $S$ es definido por la siguiente expresión:

$$
S=\left(\frac{25.400}{C N}\right)-254
$$

Donde: $\mathrm{CN}$ es el Curve Number (Número de Curva de escorrentía superficial) que corresponde a un número adimensional determinado por experimento en diversas cuencas, que va de 0 a $100(0 \leq C N \leq 100)$. Las superficies impermeables y cuerpos de agua poseen un $C N$ igual 100, mientras que superficies naturales tienen un $C N$ menor a 100. Cada cobertura de suelo tiene un $C N$ particular, y el valor $S$ final de la cuenca resulta de una sumatoria ponderada de todos los $C N$ parciales. En el caso que la precipitación de tormenta sea menor a 0,2 por $S$, la escorrentía será cero (Zhan y Huang, 2004).

El modelo TR 55 ha tabulado un Número de Curva para condiciones antecedentes de humedad normales (AMC II), condiciones secas (AMC I) y condiciones húmedas (AMC III), de acuerdo a dos aspectos: el grupo hidrológico del suelo, el cual depende del tipo de drenaje del suelo; y el uso/cobertura de suelo, que se explicará más adelante. Respecto al grupo hidrológico del suelo, el SCS ha definido cuatro grupos de suelos a partir de sus distintas tasas de infiltración, que van de la más baja escorrentía potencial (Grupo A) a la más alta (Grupo D). Para ello, se adaptaron a la clasificación del SCS los tipos de suelos obtenidos de las ortofotos de CIREN (Centro de Información de Recursos Naturales), escala 1:20.000, de la comuna de Chillán y Chillán 
Viejo considerando las características de drenaje de ellos (Cuadro 2). Para las comunas de Pinto, Coihueco y San Ignacio se consultó la información de cada serie de suelo que se encuentra disponible libremente en la página web del Sistema de Información Territorial SIT del CIREN, http://sit.ciren.cl/, y luego se revisó el tipo de drenaje de cada serie de suelo en los estudios Agrológicos de la VIII Región del Biobío (CIREN, 1996, 1999), información que se ingresó a la cobertura digital en ArcGIS.

\section{Cuadro 2}

ADAPTACIÓN DE LAS SERIES DE SUELOS DE CIREN AL SISTEMA DE GRUPO HIDROLÓGICO DEL SUELO DEL TR 55

\begin{tabular}{lc}
\hline $\begin{array}{c}\text { Tipo de drenaje de las series } \\
\text { de suelo del CIREN }\end{array}$ & $\begin{array}{c}\text { Grupo hidrológico del suelo } \\
\text { del TR 55 }\end{array}$ \\
\hline 1, muy pobre & Grupo D \\
2, pobre & Grupo D \\
3, imperfecto & Grupo C \\
4, moderado & Grupo B \\
5, bueno & Grupo A \\
6, excesivo & Grupo D \\
\hline
\end{tabular}

Nota: Es importante mencionar que la parte alta de la cuenca, Cordillera de Los Andes, no dispone de información de drenaje de suelos. Para efectos de calcular el CN ponderado de la cuenca se consideró como una unidad homogénea asignándola al Grupo D, en razón de que las coberturas predominantes son suelo desnudo, nieve y bosque nativo.

El siguiente paso fue asignar y adaptar el $C N$ de cada uso/cobertura de suelo según grupo hidrológico, de acuerdo al porcentaje de impermeabilización para el uso urbano y condiciones hidrológicas para el resto de los usos. Para ello, se superpusieron por medio del SIG, los planos de tipo de suelo hidrológico con los planos de uso/cobertura de suelo para los distintos años en análisis (1976, 1989, 1999 y 2007). Luego se consultó las tablas de CN del USDA para adaptar el valor de CN según el tipo de uso de suelo identificado.

Luego para calcular la escorrentía potencial se asumió eventos de precipitación máximas en 24 horas uniformes de 20 a $120 \mathrm{~mm}$, con un incremento constante de $10 \mathrm{~mm}$. Finalmente, para caracterizar episodio de precipitaciones extremas reales se emplearon datos correspondientes a registros meteorológicos de la Facultad de Ingeniería Agrícola de la Universidad de Concepción 
para el período 1969-2006. También se recopiló información de estaciones hidrológicas de la Dirección General de Aguas (DGA), antecedentes sobre inundaciones del Ministerio de Vivienda y Urbanismo (MINVU), Ministerio de Obras Públicas (MOP) y la Oficina Nacional de Emergencia (ONEMI).

\section{RESUlTAdOS}

\section{Cambio de uso de suelo en la cuenca}

Dentro de las distintas categorías de usos de suelo identificadas en la cuenca del río Chillán se puede determinar que en estos más de treinta años el uso matorral y pastizal es el más predominante, representado sobre el $27 \%$ del uso de la cuenca al año 2007 (figura 2). A continuación viene el uso de bosque nativo y terrenos agrícolas que en conjunto se reparten el 50\%. Los usos urbanos y plantaciones forestales si bien no destacan por su extensión, si lo hacen respecto al gran crecimiento que han experimentado entre los años 1976 y 2007 ; de un 1,3 a 2,4\% y de 1,4 a $13,3 \%$, respectivamente.

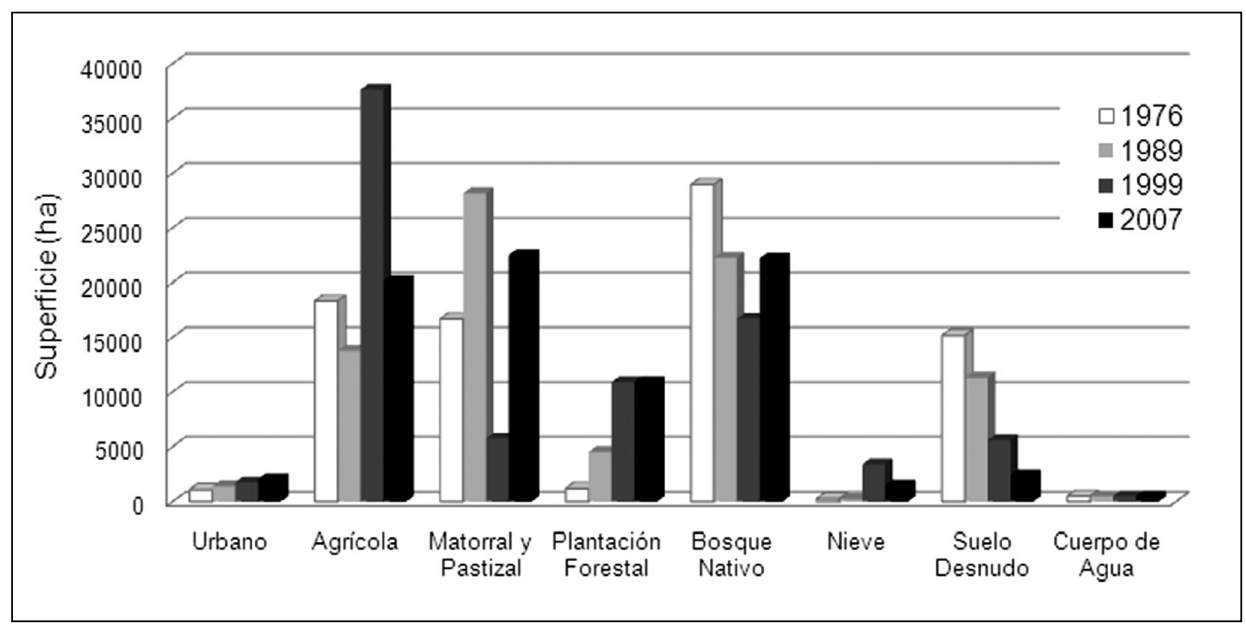

Figura 2. Evolución de la superficie de usos de suelo de la Cuenca de Chillán entre 1976-2007

En el caso urbano, representado por la ciudad de Chillán Viejo, parte de Chillán, localidad de Pinto y el sector periurbano ubicado entre Chillán y 
Pinto, se puede afirmar que ha sido una cobertura con un crecimiento constante en el tiempo y probablemente siga esa tendencia. Es importante destacar que el crecimiento urbano se encuentra asociado a la disponibilidad de equipamientos, comercio y servicios que ofrece la ciudad de Chillán y que presenta un carácter fuertemente fragmentado debido al despliegue importante de parcelas de agrado, condominios cerrados y loteos unifamiliares en el espacio periurbano. Mayores antecedentes de esta dinámica se puede consultar en Henríquez et al (2006).

La dinámica de expansión de plantaciones forestales también exhibe un interesante despliegue en la cuenca, ligado a factores económicos, políticos y naturales que explican su acelerado crecimiento (Henríquez, 2008). En términos espaciales existen tres patrones; el primero, de mayor extensión, se distribuye en la depresión intermedia al sur del río Chillán, en su sección media, donde se presentan grandes paños de bosque de pino radiata. El segundo se concentra en la precordillera andina, planteando un conflicto de uso entre la pérdida de bosque nativo por avance altitudinal de las plantaciones forestales, donde predominan tupidos bosques de Nothofagus hasta aproximadamente los $1.800 \mathrm{~m}$ de altitud (Mardones et al, 2001). Finalmente, el tercer foco incipiente, surgido en esta última década, es el que se presenta en la parte inferior de la cuenca, ligado a la influencia territorial que ha ejercido el Complejo Forestal Itata sobre la cuenca del Ñuble.

Las categorías suelo desnudo junto con las coberturas de nieve y cuerpos de agua, si bien muestran fluctuaciones entre los distintos años, se pueden considerar como coberturas relativamente constantes ya que en su dinámica temporo-espacial no participan factores socio-económicos que expliquen sus cambios.

\section{Efectos de la urbanización de la cuenca sobre la escorrentía superficial}

Para evaluar el comportamiento de la escorrentía superficial en función de la precipitación de tormenta se construyeron curvas que relacionan ambas variables para los años en análisis. En la figura 3 se puede observar que sobre los $20 \mathrm{~mm}$ de precipitación comienza a generarse escorrentía superficial de manera creciente a medida que la precipitación incrementa su monto, hasta llegar a los $120 \mathrm{~mm}$ de agua caída. Es interesante notar que no hay un aumento temporal de los montos de escorrentía, en efecto las profundidades de escorrentía estimadas para los años 1999 y 1989 son superiores al año 2007, esto se debe al efecto de infiltración de los suelos donde se extienden los bosques, especialmente las plantaciones de pino radiata. 


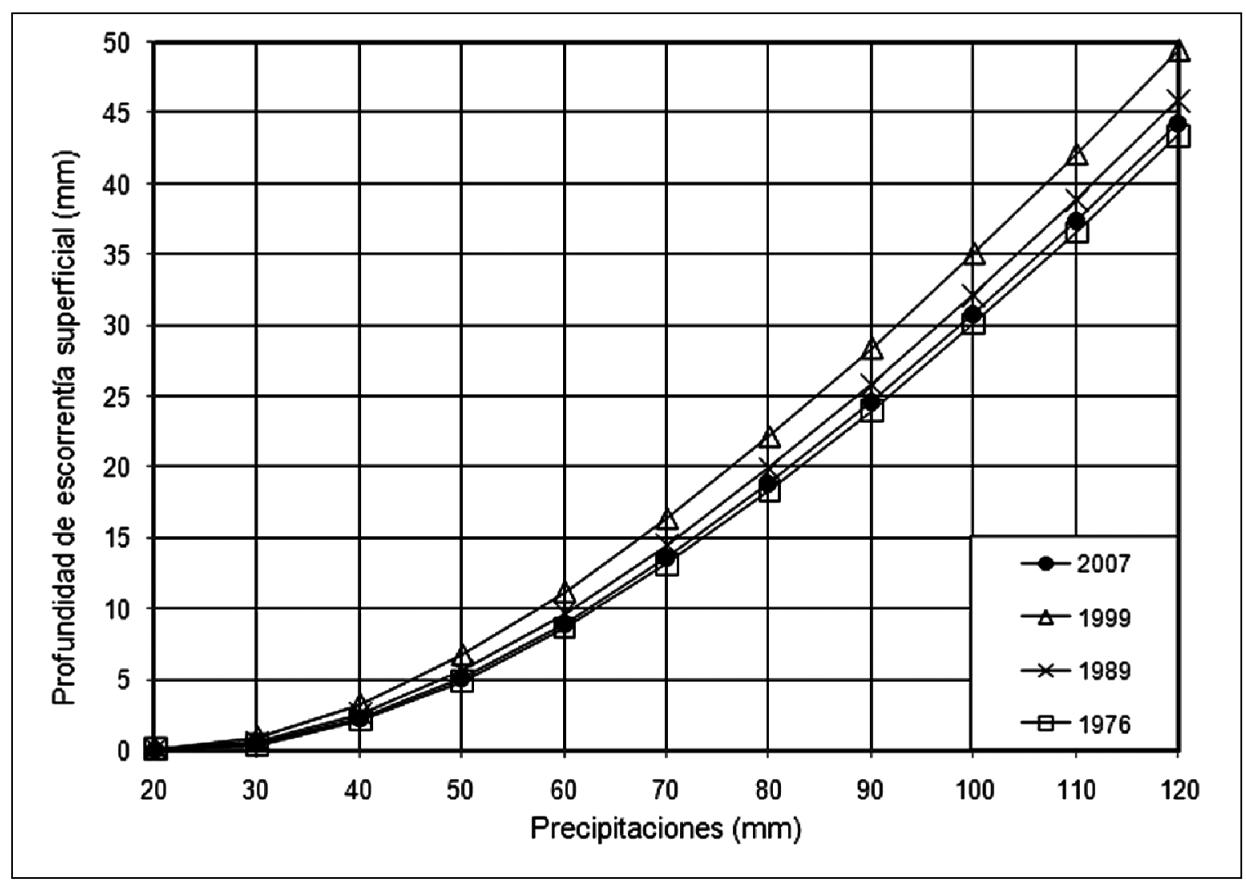

FIgURA 3. Estimación de la escorrentía potencial en la Cuenca de Chillán

Estos resultados representan al comportamiento global de la cuenca y deben interpretarse en esa escala de análisis; esto implica que en una precipitación de tormenta de $100 \mathrm{~mm}$, aproximadamente el 30\% escurre en superficie y el resto se infiltra, lo cual puede resultar no tan importante como la situación que se presenta al interior del área urbana, donde el proceso de impermeabilización es más intenso, generando importantes perjuicios debido a inundaciones y anegamientos.

Según lo planteado por Henríquez et al (2006) es importante destacar que con una adecuada planificación del territorio a nivel de cuencas urbanizadas es posible mantener e incluso aminorar los efectos derivados de la impermeabilización de suelos y el aumento de la escorrentía superficial, a través de una ordenación armónica del territorio, por ejemplo, respetando ciertas densidades de ocupación del suelo en áreas de borde con capacidad de retención de humedad o bien manteniendo los parches de vegetación natural en zonas húmedas o cursos de agua. De este modo, y desde una perspectiva ambiental, será posible un proceso de crecimiento urbano más sostenible, en la medida 
que los nuevos usos del suelo y densidades de ocupación, permitan o favorezcan una mayor capacidad de infiltración del agua lluvia y, por otro lado, morigeren el escurrimiento superficial y sus efectos sobre la ciudad.

\section{Comportamiento de las precipitaciones extremas}

Los datos de precipitaciones máximas en 24 horas para el período 19692006 referido a la estación del campus de la Universidad de Concepción ubicada al interior de la ciudad, muestran que el año 1983 fue el año de mayor monto, donde se registró $148,7 \mathrm{~mm}$ de agua caída en 24 horas, no obstante el año más lluvioso correspondió al año anterior, con $1.616 \mathrm{~mm}$. La variabilidad interanual de las precipitaciones, característica de la zona mediterránea, tiene un correlato con el fenómeno ENSO (El Niño Oscilación del Sur), en el sentido de relacionarse con los años más lluviosos (figura 4).

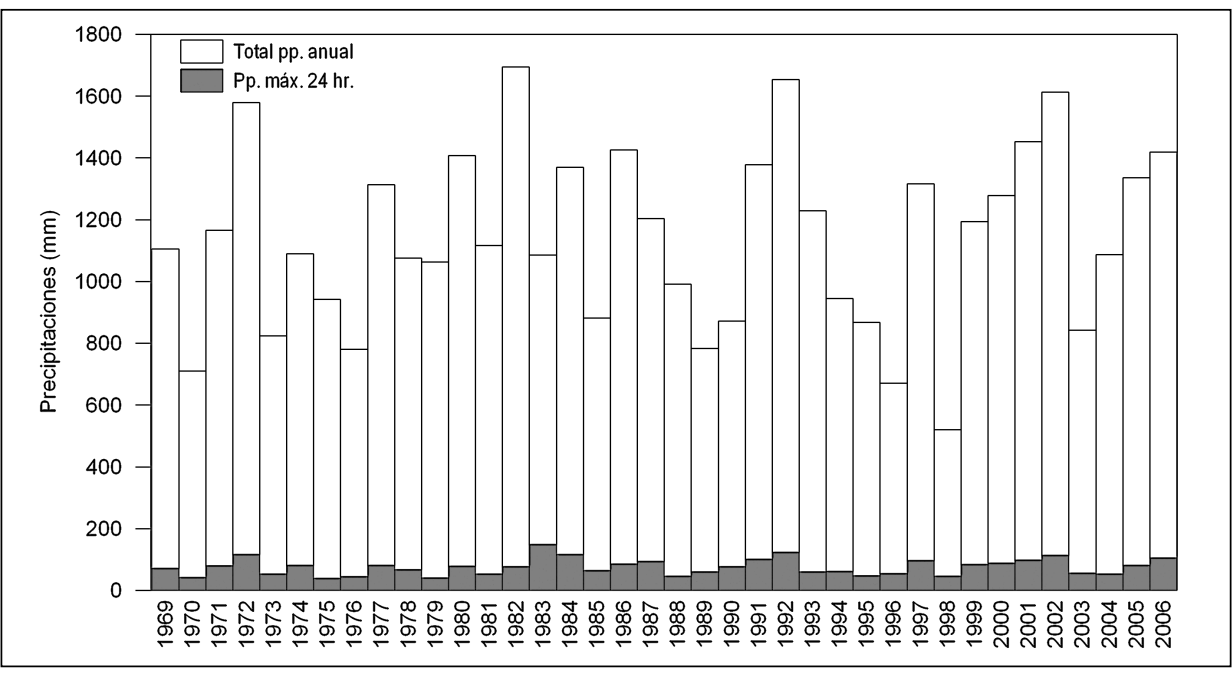

Fuente: Facultad de Ingeniería Agrícola (UDEC, 2007).Estación Campus Chillán

FIGURA 4: Precipitaciones anuales y precipitaciones máximas en 24 horas, Estación Campus Chillán

El promedio de precipitaciones máximas en 24 horas para el período 19692006 es de 76,7 mm y la desviación estándar 26,39 mm. Mientras que la fre- 
cuencia de precipitación máxima diaria, según intervalos de intensidad para el período, indica que los valores entre 80 a $90 \mathrm{~mm}$ en 24 horas concentran el mayor número de casos ( 7 casos), junto con el rango de 40-50 mm con igual cantidad.

Ahora bien, si se analizan las precipitaciones máximas en 48 y 72 horas, se puede establecer que la precipitación máxima en 48 horas corresponde al año 1992 con $240 \mathrm{~mm}$ de agua caída. Y en 72 horas al mismo año con 328,8 mm, entre los días 3-5 de mayo de 1992. Le siguen de manera descendente los años 2001 (201,8 mm), $1974(200 \mathrm{~mm}), 1991(192,1 \mathrm{~mm})$ y $1983(187 \mathrm{~mm})$. Valores en conjunto muy superiores a los valores estimados para calcular la escorrentía potencial.

\section{Caracterización del sistema frontal del 10-11 de julio de 2006 y daños asociados}

El año 2006 fue un año lluvioso registrándose 1.312,8 mm, superando en $262 \mathrm{~mm}$ a un año normal $(1,050 \mathrm{~mm})$. De acuerdo al Boletín Climático del Departamento de Geofísica (DGF) de la Universidad de Chile, el mes de julio de 2006 fue un mes particularmente lluvioso, registrándose una anomalía de precipitación superior a $100 \mathrm{~mm}$.

La precipitación de tormenta del 10 al 11 de julio de 2006, duró 34 horas continuas, aportando 40,9 $\mathrm{mm}$ el primer día y 116,2 $\mathrm{mm}$ el segundo, originándose graves inundaciones, anegamientos y desbordes de ríos, esteros y canales en la ciudad de Chillán.

El evento comienza suavemente la tarde del lunes de 10 de julio a las 15:00 horas con 0,12 $\mathrm{mm}$, aumentando su intensidad hasta llegar a un peak a las 22:00 horas $(7,9 \mathrm{~mm})$; luego comienza a descender hasta las 5:00 horas; posteriormente, comienza a incrementar la intensidad hasta llegar a un peak a las 18:00 horas con 15,91 mm, para luego retraerse, finalizando en la noche del día 12 de julio. En total precipitan 157,1 mm, que representa el $8 \%$ del monto pluviométrico anual y casi el $50 \%$ de la precipitación de julio.

Los días previos también había precipitado, alcanzando entre el día 6 al 9 de julio a $52,94 \mathrm{~mm}$, saturando de esta forma el suelo y explicando de alguna forma las consecuencias de desbordes en esteros y canales que acompañaron al fenómeno meteorológico del 11 de julio. Desde fines de mayo y hasta la tercera semana de julio la actividad frontal fue prácticamente constante en la región; durante julio se registraron cuatro episodios, destacando 
el ocurrido entre el 5 al 12 de julio que estuvo acompañado por masas de aire cálido y temperaturas mínimas relativamente altas (DGF, 2007). Este sistema frontal afectó a prácticamente todo Chile Central, siendo la VIII región la más afectada.

En lo que respecta a la ciudad de Chillán y de acuerdo a los antecedentes aportados por ONEMI (ONEMI, 2007) se registraron un total 10.000 personas damnificadas en la comuna, de un total regional de 51.206 personas. Por su parte, en Chillán Viejo se registraron sólo 5 personas damnificadas.

De acuerdo a antecedentes aportados por el diario La Discusión de Chillán se registraron dos víctimas fatales: una en el sector del callejón Bustamante, en el kilómetro 15 del camino a Pinto al ceder el puente El Esfuerzo que cruza el canal Cada Cada; y la segunda, en el estero Los Camarones, ubicado entre avenida Los Puelches y calle Cerro Roble al interior de la ciudad de Chillán (Discusión, 2006).

En cuanto a las viviendas involucradas, Chillán Viejo registró una vivienda destruida, mientras que en Chillán el total de residencias dañadas se distribuyó en las tres categorías empleadas por ONEMI, de la siguiente forma: 2.100 con daño menor; 150 con daño mayor y 30 viviendas destruidas. Los daños registrados sobre la infraestructura vial se concentraron en 25 puntos críticos dentro de la región, de los cuales ninguno se localizó en las comunas que comprenden el área de estudio. Tampoco se produjeron aluviones o derrumbes de importancia, exceptuando el dramático aluvión de Chiguayante que cobró 10 víctimas fatales.

Respecto a la distribución espacial de los principales puntos y áreas de inundación en la ciudad levantadas por el Municipio y Secretaría Regional del Ministerio de Vivienda y Urbanismo, luego del paso del frente, se puede observar una concordancia con las áreas de inundación identificadas en el Plan Maestro de Aguas Lluvias (INGENDESA, 2002).

En resumen este evento ha sido uno de los más desastrosos de las últimas décadas aunque no ha sido el más intenso. Los sectores más afectados corresponden al estero Las Toscas y en especial los canales que cruzan la ciudad (Huambalí, la Luz de Cato, entre otros), los cuales colapsaron e inundaron grandes sectores urbanos, al igual que el sistema de evacuación de aguas lluvias y sectores con topografía baja al sur de la ciudad. Los efectos acaecidos permiten plantear; ¿hay instrumentos que permitan prevenir o disminuir estos impactos?, ¿las actuales tendencias de urbanización reconocen las limitaciones impuestas por las dinámicas naturales? 
EL ROL DE LA PLANIFICACIÓN TERRITORIAL EN LA CAPACIDAD ADAPTATIVA DE LA CUENCA

La planificación territorial juega una importante función como instrumento preventivo de eventos asociados a precipitaciones extremas. La definición de zonas de extensión en urbana en suelos con buena capacidad de infiltración y su posterior consolidación puede aumentar sustantivamente los coeficientes de escorrentía y con ello inundar y anegar sitios que antes no eran afectados.

En este sentido resulta interesante cotejar la zonificación del reciente Plan Regulador Intercomunal Chillán-Chillán Viejo, aprobado el año 2007, con los estudios de riesgo de inundación encargados por el Ministerio de Obras Públicas mencionados anteriormente. Destacando lo siguiente (PRISMA, 2004):

- Las zonas de inundación se concentran principalmente en los cauces abandonados del río Chillán o paleocauces como río Viejo al sur de Chillán Viejo.

- Para la mayoría de estas zonas se proponen zonas de restricción como la zona de protección de drenaje (ZPD).

- En algunos sectores bajo riesgo de inundación, como en las proximidades del puente El Saque, se proponen zonas habitacionales mixtas (ZHM2) que permiten densidades medias con coeficientes máximos de ocupación de suelo de 0,8 .

- En torno al río Chillán se propone la Zona Residencial de Parcelas (ZRP) que corresponden a zonas que sirven de interfase o transición entre las áreas urbanas y las áreas rurales intercomunales, y cuyo destino preferente es el habitacional, y equipamiento complementario. Se proponen coeficientes de ocupación de 0,1 que resultan más apropiados para disminuir la escorrentía, ya que aseguran una baja impermeabilización del suelo, especialmente en sectores como El Emboque donde existe una potencial zona inundable.

Para estos sectores el estudio de PRISMA recomienda, que por tratarse de terrenos bajos su urbanización requeriría de importantes rellenos y de la construcción de obras de defensa fluvial. Esta situación se ve agravada por la existencia de bocatomas de los canales San Juan y San Bernardo que operan de forma artesanal.

Este tipo de intervenciones para disminuir la vulnerabilidad plantean a modo de conclusión muchas incertidumbres sobre la real efectividad de una planificación proactiva y no reactiva. Una planificación que apuesta a la miti- 
gación y no a la prevención de riesgos naturales es mucho menos sostenible que una planificación basada en los conceptos de resiliencia y sobre todo en la capacidad adaptativa (Gallopín, 2006). Al parecer no se han aprendido las lecciones de un pasado marcado por desastres naturales.

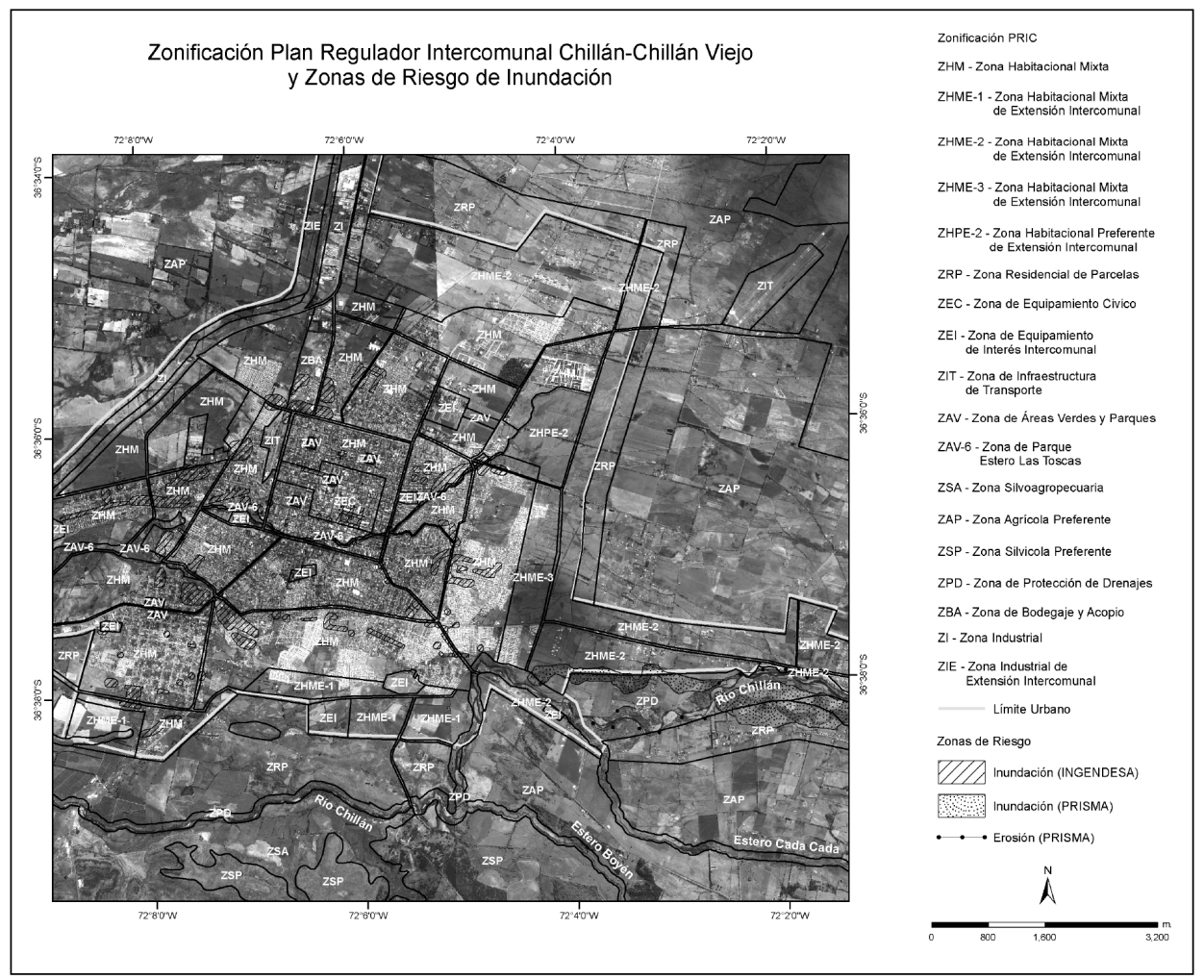

Figura 5. Relación entre la zonificación del Plan Regulador Intercomunal con las Zonas de riesgo de inundación

El ejemplo de Chillán con sus cuatro fundaciones históricas y múltiples reconstrucciones contemporáneas, al igual que otras ciudades como Concepción que también tuvo que relocalizarse por la destrucción de un tsunami o el caso reciente de Chaitén arrasado por la erupción del volcán homónimo y posterior inundación, plantea la urgente necesidad de cambiar el modo de planificar y gestionar nuestras ciudades. 
Históricamente en la cuenca de Chillán se han registrado montos de precipitaciones máximas en 24 horas similares o mayores al del día 11 de julio de 2006, sin embargo no se habían registrado daños tan graves como los de ese episodio, en otras palabras no se ha logrado una capacidad adaptativa. Esto quiere decir que iante un fenómeno hidrometeorológico excepcional o incluso normal se está haciendo más vulnerable la ciudad? Evidencias en ciudades chilenas, como por ejemplo Concepción (Mardones, Echeverría y Jara, 2004) y argentinas, como por ejemplo la ciudad de Pergamino (Celis, et al., 2005), permite afirmar que el impacto de las inundaciones ha aumentado frente a una misma intensidad de lluvia.

Sin duda los factores locales ayudan a entender la intensificación de los efectos de precipitaciones extremas, como la impermeabilización de zonas sensibles o pérdida de coberturas vegetales de la cuenca. No obstante también aparecen factores globales como el calentamiento global, lo cual exige abordar un problema complejo cuya solución pasa por ampliar la escala de análisis y reconocer a la cuenca urbana como una unidad de planificación territorial, a la cual deben estar dirigidas todas las medidas preventivas y de mitigación de manera integrada y participativa. Junto con ello es de especial relevancia contar con un sistema de predicción y alerta hidrológica que mejore la capacidad adaptativa de la cuenca.

Estas son algunas de las principales tareas que debe abordar la planificación territorial chilena frente al desafío que impone un Bicentenario más sostenible.

Recibido: 18/02/2009

Aceptado: 03/03/2009

\section{BibLiografíA}

Adger, W. N., Hughes, T. P., Folke, C., Carpenter, S. R. y Rockström, J. (2005): Socialecological resilience to coastal disasters. Science, 308(5737), pp. 1036-1039.

Aldana, A. (1998): Sistemas de ayuda a la explotación de un SAIH. Madrid: Centro de Estudios y Experimentación de Obras Públicas. Ministerio de Fomento.

Asta-Buruaga, F. (1899): Diccionario Geográfico de la República de Chile (2ª ed.). Santiago: Brockhaus.

Brand, F. S. y Jax, K. (2007): Focusing the meanings(s) of resilience: resilience as a descriptive concept and a boundary object. Ecology and Society, 12(1), pp. 23.

Celis, A., Caputo, G., Bartolomé, M., Kisilevsky, G., Herzar, H. y Rodríguez, C. (2005): Primeros pasos hacia una política local que gestione el riesgo de inundación. Medio Ambiente y Urbanización, 62(1-2), pp. 161-174. 
CIREN (1996): Estudio de Suelos Agrológicos VIII Region, escala 1:20.000. Servidor de mapas de la Estrategia de Desarrollo Territorial Consultado el 3 de marzo, 2008.

CIREN (1999): Estudio Agrológico VIII Región. Descripciones de suelos: Materiales y Símbolos. Santiago: Centro de Información de Recursos Naturales.

Concha, M. (1981): A cuatro siglos de la fundación de Chillán. Revista de Geografía Norte Grande, 25, pp. 5-21.

Chow, V. T., Maidment, D. y Mays, L. (1994): Hidrología Aplicada (Saldarriaga, J., Trans. Edición en español ed.). Santafé de Bogotá: McGraw-Hill Interamericana, S.A.

DGF (2007): Boletín Climático Consultado el 7 de julio, 2007, http://met.dgf.uchile.cl/clima/.

Discusión (2006, 13 de julio): Dos víctimas fatales cobró frente de mal tiempo en Chillán y Pinto. Diario La Discusión. http://www.diarioladiscusion.cl/.

Gallopín, G. (2006): Linkages between vulnerability, resilience, and adaptative capacity. Global Environmental Change, 16, pp. 293-303.

GORE-BIOBIO (2007): Plan Regulador Intercomunal Chillán-Chillán Viejo. Concepción: Gobierno Regional del Biobío.

Henríquez, C. (2008): Apertura neoliberal y expansión forestal: algunos factores para su modelación en la cuenca de Chillán. Paper presentado en Actas del X Coloquio Internacional de Geocrítica. Diez años de cambios en el Mundo, en la Geografía y en las Ciencias Sociales, 1999-2008. http://www.ub.es/geocrit/-xcol/198.htm

Henriquez, C., Azocar, G. y Aguayo, M. (2006): Land use change and superficial water flows: Application of a spatial simulation model in Los Angeles, VIII Region of Biobio, Chile. Cambio de uso del suelo y escorrentia superficial: Aplicacion de un modelo de simulacion espacial en Los Angeles, VIII Region del Biobio, Chile, (36), pp. 61.

Henríquez, C., Azócar, G. y Aguayo, M. (2006): Cambio de uso del suelo y escorrentía superficial: aplicación de un modelo de simulación espacial en Los Ángeles, VIII Región del Biobío, Chile. Revista de Geografía Norte Grande, 36, pp.

Holling, C. S. (1973): Resilience and stability of ecological systems. Annual Review of Ecology and Systematics (4), pp. 1-23.

INGENDESA (2002): Plan Maestro de Evacuación y Drenaje de Aguas Lluvias de Chillán. Santiago: INGENDESA.

Mardones, M. (1990): Los riesgos naturales en el sitio de la ciudad de Chillán: Riesgo sísmico, de inundación y de anegamiento. En: Olave, D. (Ed.), Ciudades Intermedias: Chillán (pp. 8-38). Chillán: Universidad de La Serena, Facultad de Humanidades e Instituto Panamericano de Geografía e Historia.

Mardones, M., Echeverría, F. y Jara, C. (2005): Una contribución al estudio de los desastres naturales en Chile Centro Sur: efectos ambientales de las precipitaciones del 26 de junio del 2005 en el área Metropolitana de Concepción. Investigaciones Geográficas (38), pp. 1-25.

Mardones, M., Hernández, H., Cristi, O., Sánchez, A., Carrasco, P., Millán, J. y Peña, L. (Eds.). (2001): Geografía VIII Región del Bíobío. Santiago: Instituto Geográfico Militar (IGM). 
Melesse, A. y Wang, X. (2007): Impervious Surface Area Dynamics and Storm Runoff Response. En: Weng, Q. (Ed.), Remote Sensing of Impervious Surface (pp. 369-384). Boca Raton: Taylos \& Francis Group, LLC.

Mockus, V. (1972): Chapter 10. Estimation of direct runoff from storm rainfall. En: U.S. Departament of Agriculture, S. C. S. (Ed.), SCS National Engineering Handbook, Section 4: Hidrology (pp. 30). Washington, DC: U. S. Government Printing Office.

Niemeyer, H. y Cereceda, P. (1984): Hidrografía. Santiago: Instituto Geográfico Militar.

ONEMI (2007): Informe de análisis de riesgo, julio 2006. Santiago: Oficina Nacional de Emergencias (ONEMI).

Parra, O. (2000): Gestión Integrada de Cuencas Hidrográficas. Capítulo 1: Introducción. Curso Gestión Integrada de Cuencas Hidrográficas. Concepción: Centro EULA, Universidad de Concepción.

PRISMA (2004): Plan Maestro de Manejo de Cauces Cuenca del Río Chillán, VIII Región. Santiago: PRISMA Ingeniería Limitida.

Randolph, J. (2004): Environmental Land Use Planning and Management. Washington: Island Press.

Rebolledo, J., Abarza, C., Arriagada, V. y López, C. (2002): El proceso de periurbanización en ciudades intermedias de Chile Central: Chillán. Paper presented at the IX Jornadas Cuyanas de Geografía. La Geografía frente a lo efímero y lo permanente, Mendoza.

Reyes, M. A. (1990): Evolución y ocupación urbana de Chillán. En: Intermedias, C. (Ed.), Ciudades Intermedias. Santiago: Instituto Panamericano de Geografía e Historia.

Smith, B. y Wandel, J. (2006): Adaptation, adaptative capacity and vulnerability. Global Environmental Change, 16(3), pp. 282-292.

UDEC (2007). Datos meteorológicos. Chillán: Facultad de Ingeniería Agrícola, Campus Chillán, Universidad de Concepción.

Urrutia, R. y Lanza, C. (1993): Catástrofes en Chile 154-1992. Santiago: Editorial La Noria.

USDA (1986): Urban Hydrology for Small Watershed. TR-55, United States Department of Agriculture. Natural Resources Conservation Service. Conservation Engineering Division. Technical Release 55. Disponible en http://www.cpesc.org/reference/tr55.pdf

Walker, B., Holling, C. S., Carpenter, S. R. y Kinzig, A. (2004): Resilience, adaptability and transformability in social-ecological systems. Ecology and Society, 9(2), pp. 5.

Zhan, X. y Huang, M.-L. (2004): ArcCN-Runoff: an ArcGIS tool for generating curve number and runoff maps. Environmental Modelling \& Software, 19 (10), pp. 875-879.

\section{RESUMEN}

Chillán ha sido golpeado históricamente por fenómenos hidrometeorológicos, uno de los más recientes fue el de julio de 2006 que generó grandes daños, damnificados y víctimas fatales, lo que ha demostrado la alta vulnerabilidad y baja capacidad adapta- 
tiva de la cuenca. En esta investigación se analiza el proceso de urbanización en la cuenca de Chillán entre 1976-2007 y su relación en el cambio en la escorrentía superficial. Si bien se observa un aumento poco significativo en los montos de escorrentía estimados, las tendencias de urbanización, estudios de inundación y consecuencias de precipitaciones torrenciales, demuestran la importancia de contar con instrumentos de planificación territorial que actúen de manera preventiva y no reactiva frente a estos fenómenos.

Palabras Clave: precipitación de tormenta; escorrentía superficial; cambio de uso de suelo; Chillán.

\section{ABSTRACT}

Chillan has been historically struck by hidro-meteorogical phenomenon, one of the most recent was in july, 2006. That generated large damages, victims and others lose. This shows the high vulnerability and low adaptive capacity of the watershed.

This paper shows the process of urbanization in the basin is analyzed the period 1976-2007 in Chillan city and its relationship in the change in the surface runoff. Though a significant little increase in the likely surface runoff is observed, the tendencies of urbanization, studies of flood and consequences of torrential haste, they show the territorial planning instruments to include importance that act in a preventive way and do not revive set against these phenomena

KEY WORDS: stormwater; surface runoff; land use change; Chillán.

\section{RÉSUMÉ}

Chillán a été historiquement frappé par des phénomènes hydrométéorologiques, l'un des plus récents a été celui de juillet 2006 qui a produit de grands dégâts, des sinistrés et des morts, ce qui a démontré la haute vulnérabilité et la basse capacité adaptative du bassin-versant. Dans cette recherche on analyse le processus d'urbanisation dans le bassin de Chillán entre 1976-2007 et leur relation dans le changement dans l'écoulement superficiel.

Bien qu'on observe une augmentation peu significative dans les quantités d'écoulement prévues, les tendances d'urbanisation, les études d'inondation et les conséquences de précipitations torrentielles démontrent l'importance de disposer des instruments de planification territoriale qu'ils agissent de manière préventive et non réactive par rapport à ces phénomènes.

MoTS CLÉS: précipitation d'orage; écoulement superficiel; changement d'utilisation de sol; Chillán. 